\title{
Development of a Procedure for the Validation of Statistical Energy Analysis Simulations
}

\author{
D. Sipos ${ }^{1}$ D. Feszty ${ }^{2}$ \\ Széchenyi István University, Department of Whole Vehicle Engineering \\ Egyetem tér 1., 9026 Győr, Hungary \\ 1e-mail: sipos.david@ga.sze.hu \\ 2e-mail: daniel.feszty@audi.hu
}

Abstract: This paper describes the development of an NVH measurement procedure that can be used for comparisons to Statistical Energy Analysis (SEA). In SEA, the outputs of the simulation are ensemble averaged quantities for each subsystem, which can be obtained in measurements by averaging some measurement point results. For several reasons, the number of measurement points must be as few as possible, but at the same time, they have to provide a well approximated averaged response of the system. The sufficient number of evaluation points and excitation load cases are determined via Finite Element (FE) simulations. It is shown that in case of a simple, flat plate, 17 randomly chosen evaluation points in at least 3 load cases are enough to properly approximate the SEA results.

Keywords: SEA; NVH measurement; comparison; finite element method

\section{Introduction}

In recent years, the determination of the Noise, Vibration and Harshness (NVH) characteristics of complex structures, such as road or air vehicles via simulation methods have become one of the most important fields of technical development in the industry [1]. These structures are subjected to a wide range of excitation frequencies during their operation [2]. To calculate the low-frequency behaviour, Finite Element simulation is a commonly used tool, however, as the frequency increases, smaller elements must be used, which leads to a significant increase in the number of degrees of freedom. [3] Thus, Finite Element Method becomes prohibitive because of the excessive computational cost. Furthermore, the assumption of a deterministic behaviour for these systems is no longer valid beyond 
a certain frequency range [4]. Fortunately, there are several alternative methods for high-frequency vibro-acoustic problems, such as Statistical Energy Analysis [5].

Statistical energy analysis gives the opportunity to calculate the medium- to highfrequency dynamic response of vibro-acoustic systems. It utilizes the spatially and frequency band averaged vibrational energy over a subsystem as the primary variable of the power balance equation system, defined by [5]:

$$
\boldsymbol{P}=\omega \boldsymbol{C},
$$

In Equation (1), $\boldsymbol{P}$ and $\boldsymbol{E}$ are the vectors of injected power and the vibrational energy levels of the subsystems, respectively. The injected power is defined as a rain-on-the-roof excitation, which can be approximated as uncorrelated nodal forces. In an analogy to heat conduction, the vibrational energy can then be considered to propagate as though heat between subsystems in heat transfer. The coupling and damping coefficients are defined in matrix $\boldsymbol{C}$, while $\omega$ is the central frequency of the considered band. Figure 1 illustrates the graphical representation of an SEA model. [6]

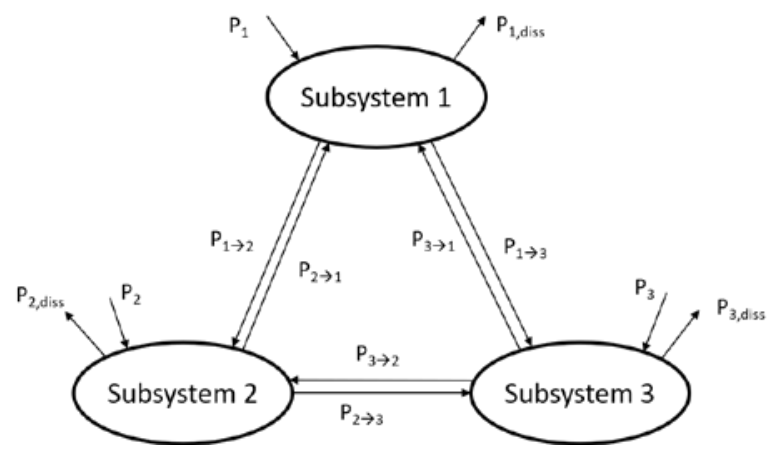

Figure 1. Representation of a SEA model [6]

Each subsystem is defined as group of similar modes, i.e. having homogeneous vibrational energy distribution in the response, or having similar damping loss factor, which must be much higher than the coupling loss factors to the other subsystems. Another key assumption of SEA is that there are enough eigenmodes in the frequency band, so that statistical behaviour of the system can be assumed [7]. This technique gives computationally affordable solutions at high frequencies, because the number of degrees of freedom are independent from the wavelength [5]. Moreover, the matrix of coefficients can be a sparse, diagonal dominated, symmetric matrix, which is well conditioned to make the calculations numerically even cheaper. 
Regarding the complex vibro-acoustic structures, numerical simulations are not yet ready to completely replace the experiments, especially in the mid- and highfrequency range. Therefore, measurements are still necessary to prove that the dynamic and acoustic behaviour of the system is as it is expected. Measurements are also used for the validation of simulations. In case of a Finite Element Analysis, it is straightforward to compare the results to experiments. Regarding the excitation in FE simulation, a point force can be applied anywhere on the structure, while in measurement, applying a shaker at the same position is equivalent. At the output points in measurements, accelerometers can be attached to the structure and then the frequency response functions are calculated by the testing software [8]. Figure 2 shows a schematic representation of a general measurement set-up.

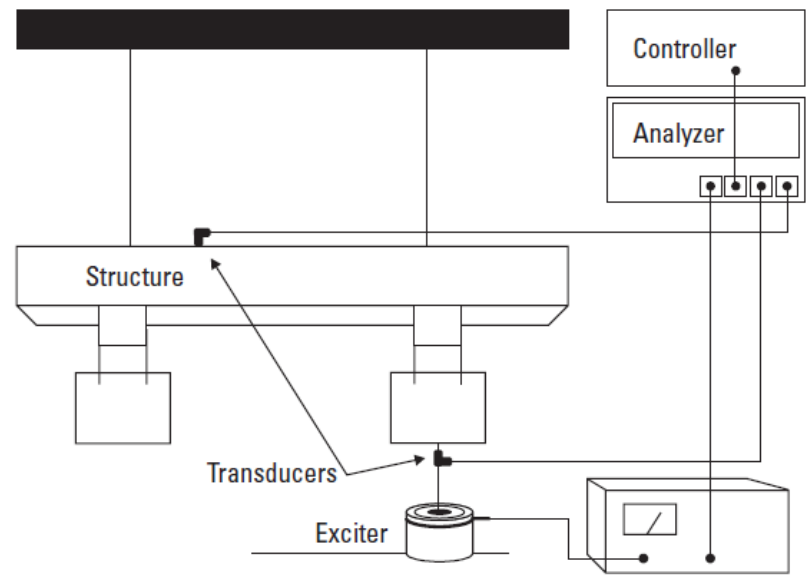

Figure 2. General test configuration [9]

However, in case of a high-frequency method like SEA, the comparison to experiments is more difficult. It is because only an averaged energy level of the subsystems is calculated, from which an averaged panel velocity is derived. As the excitation, a single point force cannot be applied at a certain position, it is distributed along the whole panel to obtain rain-on-the-roof excitation [6].

From these, it seems that some averaging of the measurement results is necessary, but there is no rule in the literature on how many outputs and load cases are required for this. In most cases, the number of the accelerometers and the measurement tools are limited, so is their availability. Furthermore, applying too many sensors at the same time could lead to high distortion in the frequency response functions, especially at higher frequencies, because of the additional mass loading [10]. 
Therefore, the goal of this paper is to propose a novel measurement procedure for validating SEA simulations. The method is demonstrated for a thin, flat plate. The aim is to determine the number of the required output points, as well as a sufficient number of load cases, from which a well approximated response of the panels can be obtained for comparison to SEA results. A special feature of the paper is, that in order to prove the concept of the measurement methodology, Finite Element simulations are used instead of real measurements, because its output is in the same format as measurement data would be.

\section{Determination of the number of required evaluation points}

The study was performed on a simple flat plate. Its dimensions were 550 x 650 $\mathrm{mm}$, with the thickness of the plate being $2 \mathrm{~mm}$. A general steel material was defined, which has the following properties:

- Young's modulus: $210000 \mathrm{MPa}$

- Poisson's coefficient: 0,3

- Density: $7850 \mathrm{~kg} / \mathrm{m}^{3}$

- Structural damping coefficient: 0,01

The plate was meshed with $10 \mathrm{~mm}$ linear quad elements. Figure 3 shows the assembled FE model; it consists of 3575 element and 3696 nodes in total. First, the sufficient number of outputs were determined, thus only one load case was defined. The excitation node was selected via random process, it is marked with black square.

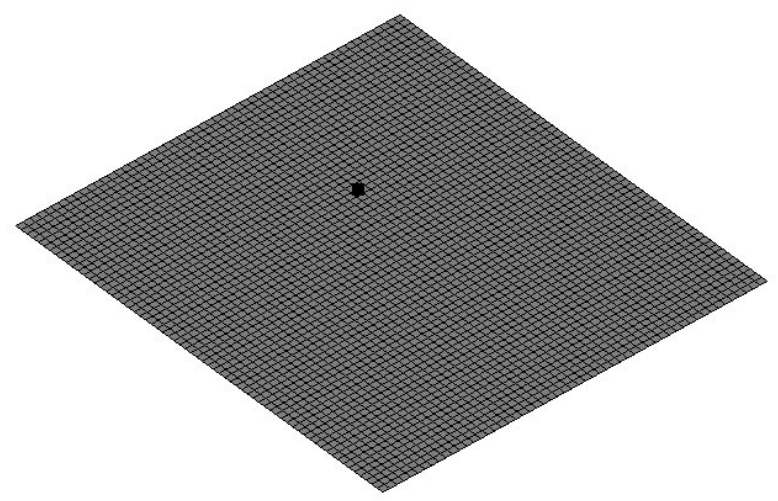

Figure 3. The finite element model, excitation node

The simulations were performed up to $1 \mathrm{kHz}$ in Virtual Performance Solution (VPS) by the ESI Group. A pre-processing script was developed, which has the 
possibility to set whether the excitation is fix or randomly selected, how many outputs are requested and whether they are randomly scattered, or are the same in each load case. First, the number of outputs was determined by the desired number of results, in this case it was 15. The sequence started from 2 and had to contain the number of all nodes, 3696. According to these criteria, a geometric series rounded to integers resulted the following numbers: $2,3,5,10,17,29,50,85,147,251,430$, $737,1262,2159,3696$. . In each case, the outputs were randomly scattered. Figure 46 show the illustration of the cases where 17, 147 and 737 output nodes were defined as examples.

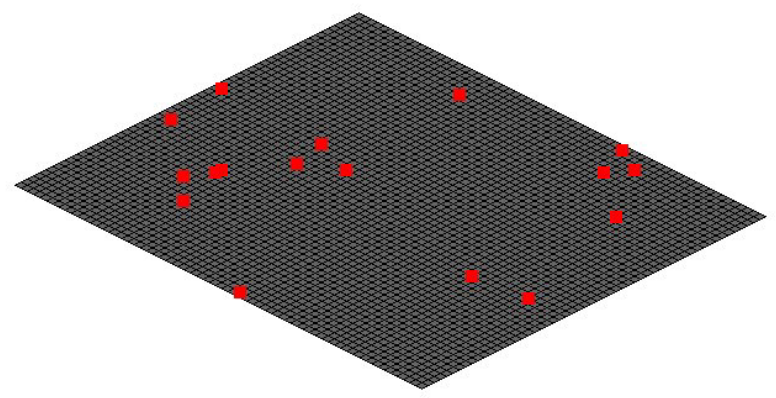

Figure 4. Finite element model, 17 output points

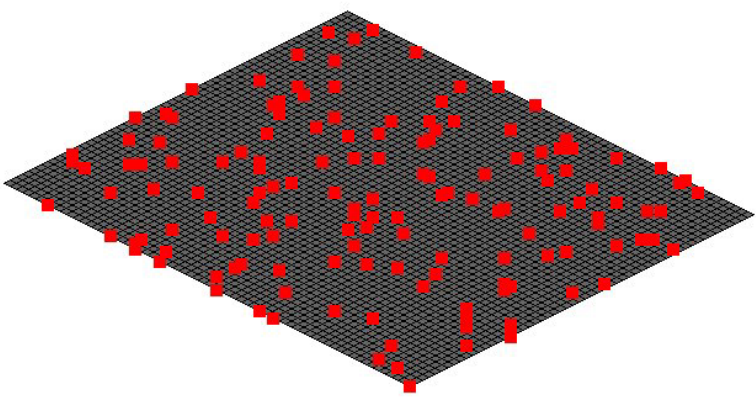

Figure 5. Finite element model, 147 output points 


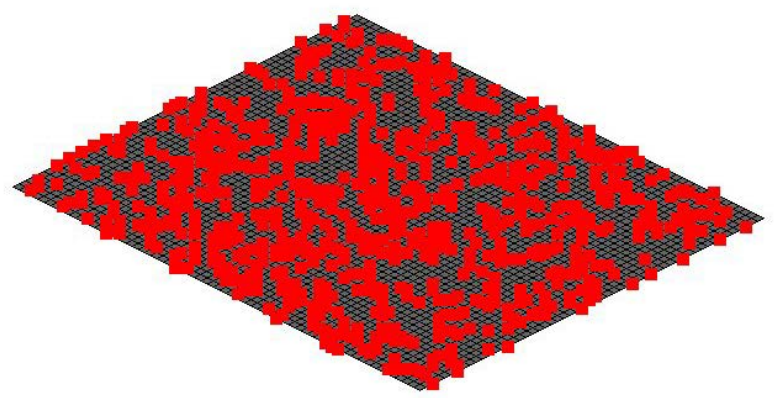

Figure 6. Finite element model, 737 output points

The phase ignored RMS velocity of the output nodes was compared to the reference, which was the RMS velocity of all the nodes, except the excitation node. Figure 7 summarizes all the results. The reference line is the black curve, the brighter the curve, the less output points are contained in the RMS velocity curve.

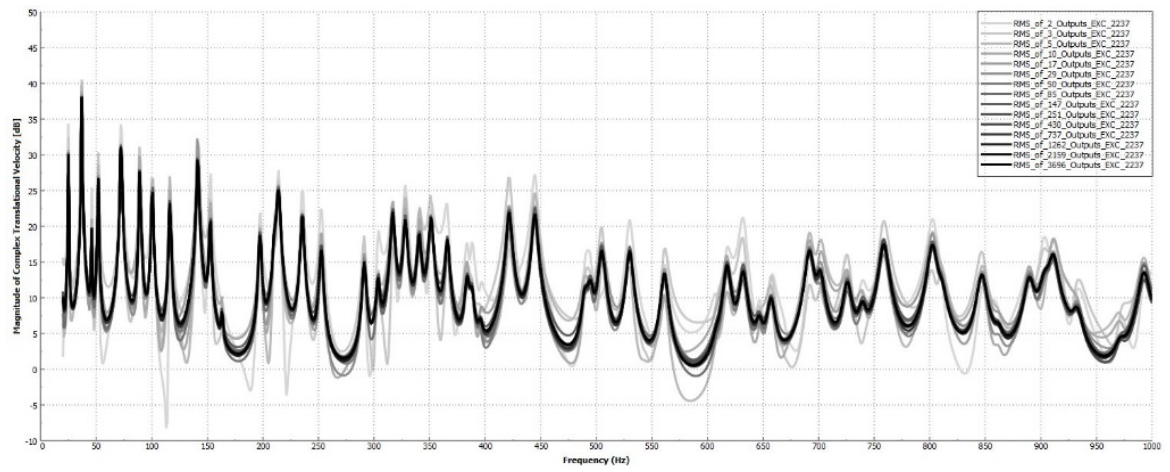

Figure 7. RMS velocities in case of different number of output nodes

It can be observed that although some discrepancies can be detected between the black and some of the brighter curves, the correlation between the darker ones is generally very good. This means that the averaged results converge to the average values of all nodes. Figure 8-10 shows the averaged results for 3, 17 and 737 nodes, respectively. 
D. Sipos and D. Feszty - Acta Technica Jaurinensis, Vol. 12, No. 4, pp. 335-346, 2019

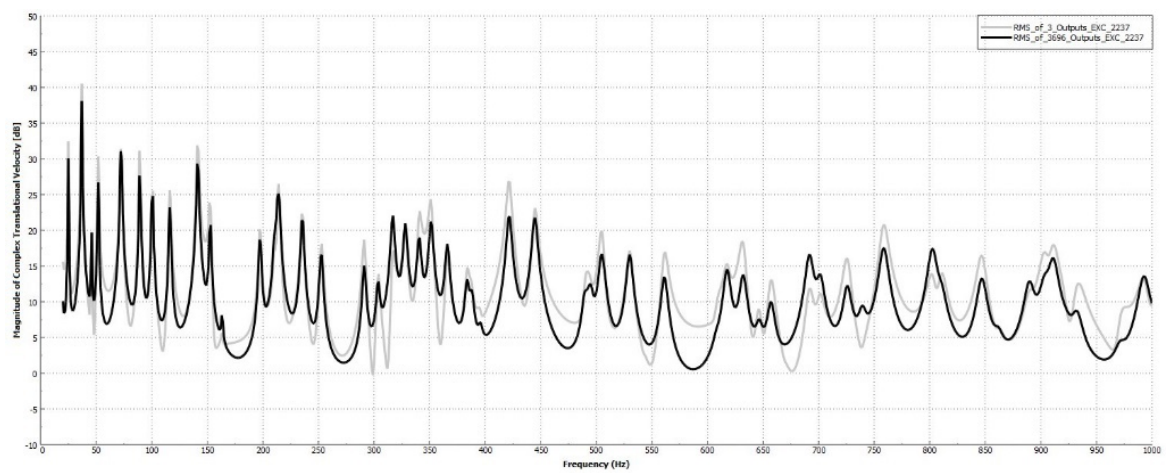

Figure 8. RMS velocity 3 nodes compared to reference

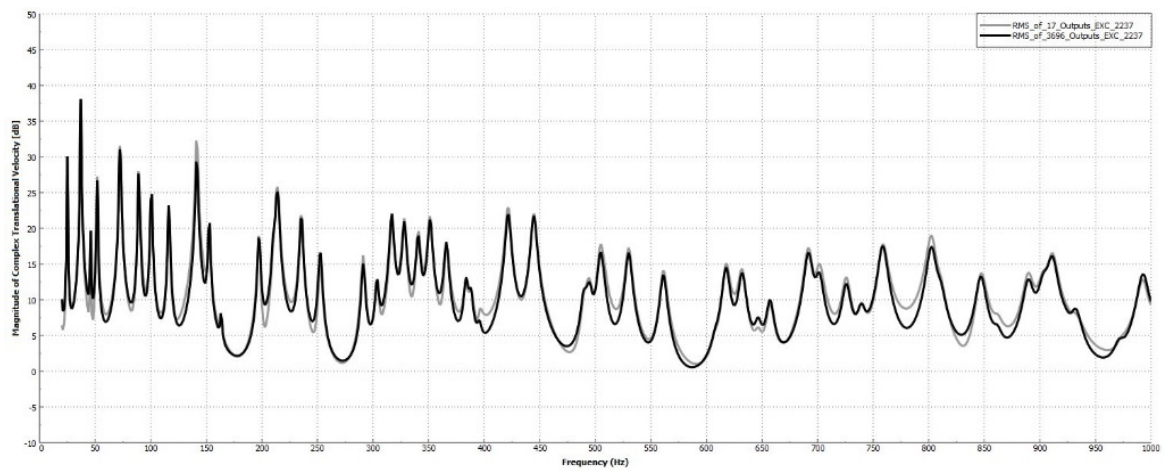

Figure 9. RMS velocity 17 nodes compared to reference

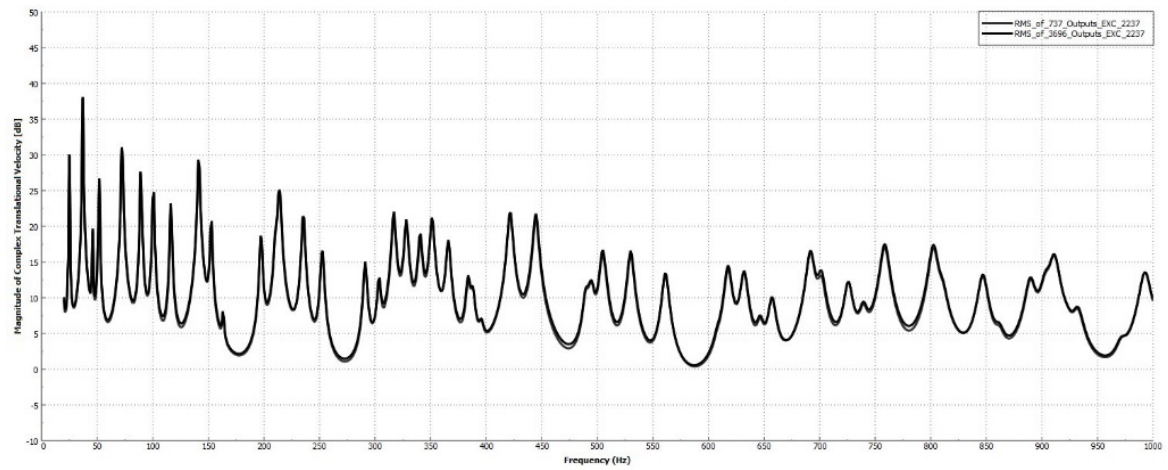

Figure 10. RMS velocity 737 nodes compared to reference 
As the results show, the averaged response of 3 randomly chosen output points has quite significant difference relative to the reference curve, but could be adequate, if only a few sensors are available. In SEA, the response of a subsystem is computed in third octave bands, so in some cases a filtered 3-node averaged result could also show acceptable correlation with SEA.

Regarding the averaged result of 737 output points, it gives almost the same curve as the all-node RMS velocity. It can be stated the results will not change beyond this number of outputs. However, 737 measurement points is still too much to realize. On the other hand the 17-nodes averaged result also shows good correlation with the reference curve, and it could be feasible in real measurements as well. It can be concluded that 3 response points are not, but 17 response points are adequate to obtain a well approximated averaged response. Note that applying 17 sensors at the same time on a plate would lead to distorted results, but in a few runs, changing the position of the accelerometers randomly is applicable.

It was also checked that the RMS velocity of 17 output nodes is independent of the position of the nodes. This is shown by Figure 11, where the RMS velocities can be seen in 8 different simulations. In each simulation, 17 different output nodes were selected, while the excitation remained in the same position.

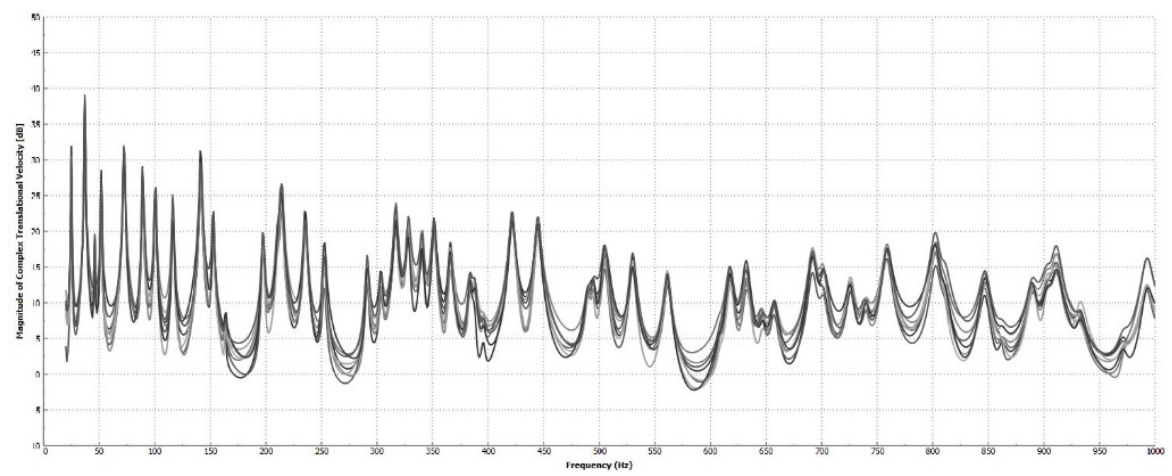

Figure 11. RMS velocities of different 17 nodes in 8 cases

\section{Effect of the excitation position}

It was proved that 17 randomly selected output points are enough to obtain a well approximated RMS panel velocity, for a given point force loading. But in SEA, the excitation is defined as a rain-on-the-roof excitation, thus it also must be checked that what effect does the position of the excitation on the averaged response of the panel. 
In 15 cases, the same 17 output nodes were selected, while the excitation position was changed randomly. The only restriction was that the excitation node must not be near the boundaries of the panel, so the outermost 3 rows of elements (the affected nodes) were excluded from the possible positions. The position of the excitation is selected from the area indicated by blue on Figure 12.

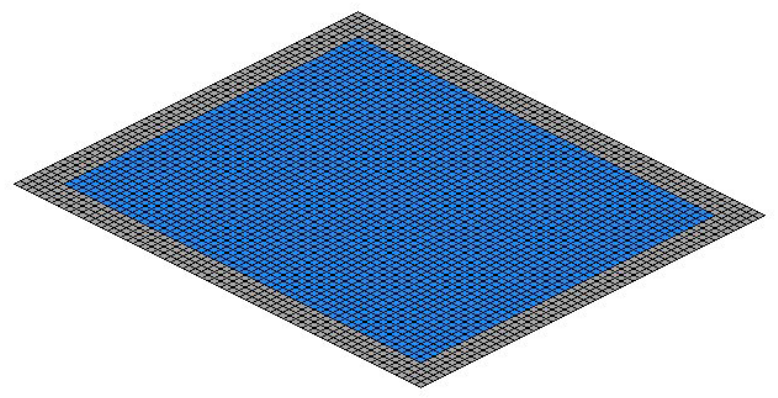

Figure 12. Area of the possible excitation location

The RMS velocities in the 15 cases is shown by Figure 13. It can be observed that between 300 and $400 \mathrm{~Hz}$, and above $600 \mathrm{~Hz}$, there are some discrepancies in the results. Taking the RMS of all the curves results in the red curve on Figure 14.

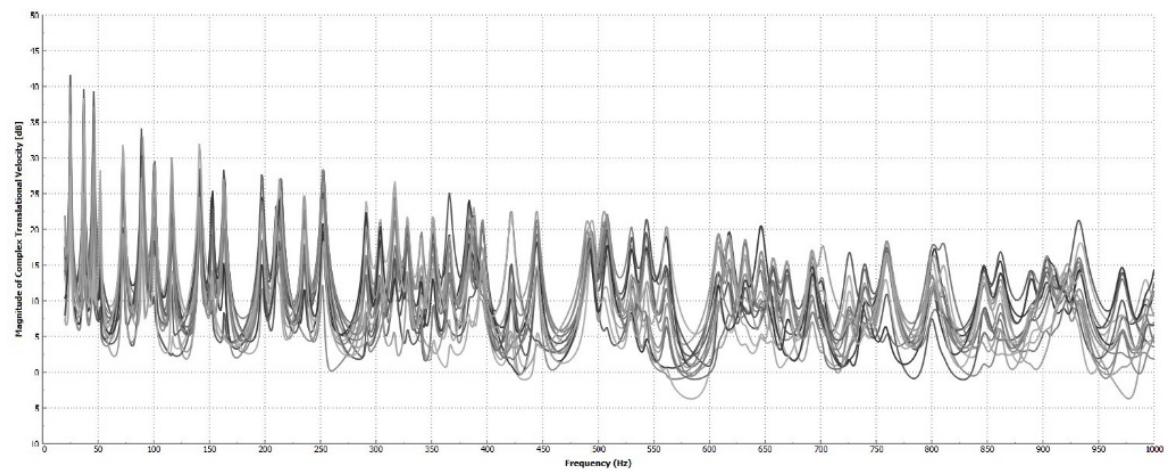

Figure 13. 17-node RMS velocities in 15 load cases 


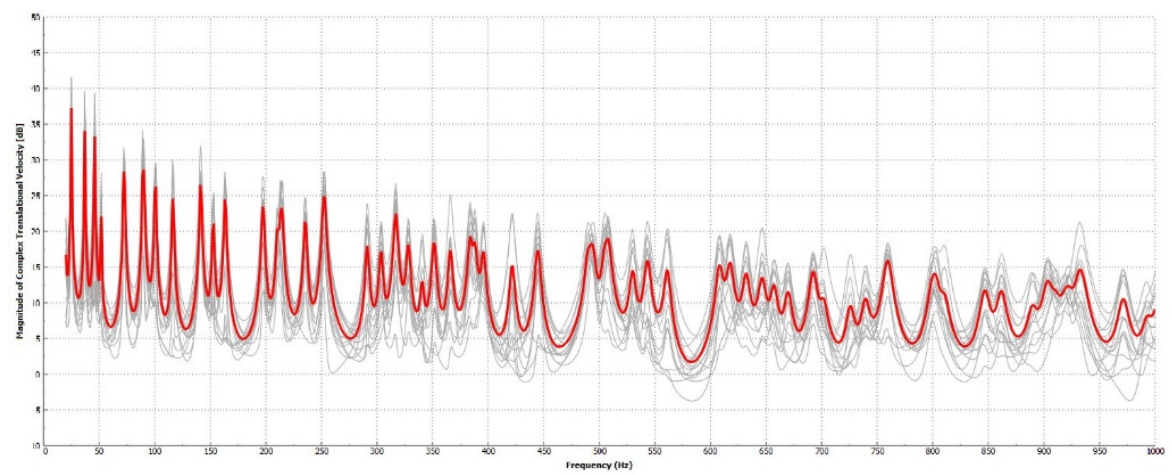

Figure 14. RMS velocity of all nodes in 15 load cases. Red line: RMS of all nodes in all load cases, grey lines: RMS of all nodes in each load cases

The RMS of all nodes in all load cases is the curve, which must be approximated in a fewer number of load cases. Taking the averages of three randomly selected curves gives the curves shown on Figure 15 by yellow, and the red one is the average of all load cases.

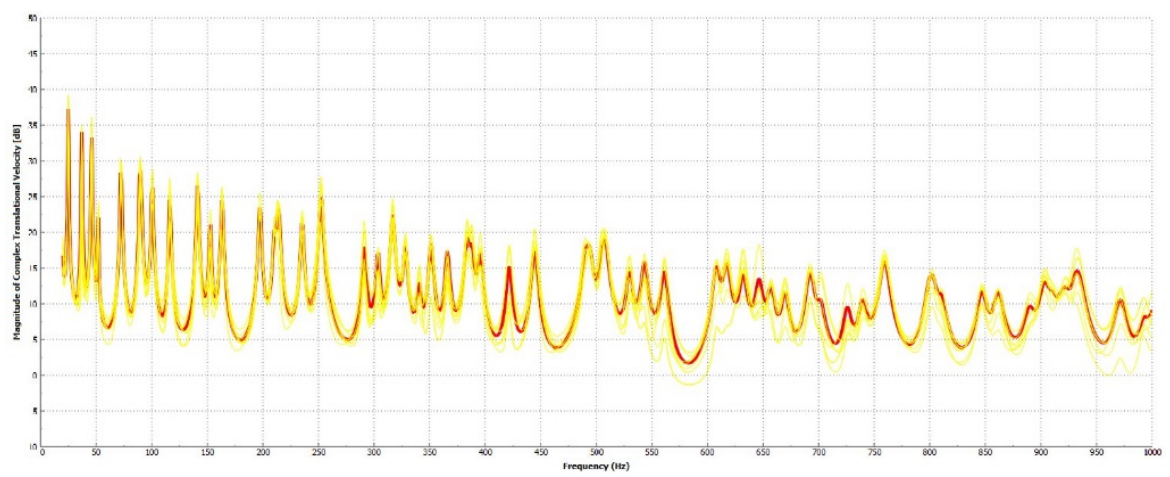

Figure 15. RMS velocity of randomly selected three load case. Red line: RMS of all nodes in all load cases, yellow lines: RMS of all nodes in 3 randomly selected load cases

Any of the yellow curves shows good correlation with the red one. It can be stated that at least 3 different load cases are enough to obtain a well approximated panel velocity, with at least 17 output nodes. 


\section{Comparison with SEA results}

To validate the above-mentioned results, a comparison to SEA was performed. The physical properties of the panel were the same as in the FE simulation. A point force of $1 \mathrm{~N}$ magnitude was defined on the plate as the excitation. The averaged panel velocity is shown on Figure 15 (by black colour). The third octave filtered, 17node, 15-load case averaged RMS velocity is indicated by red and the 17-node, 3 load case averaged result is shown by blue.

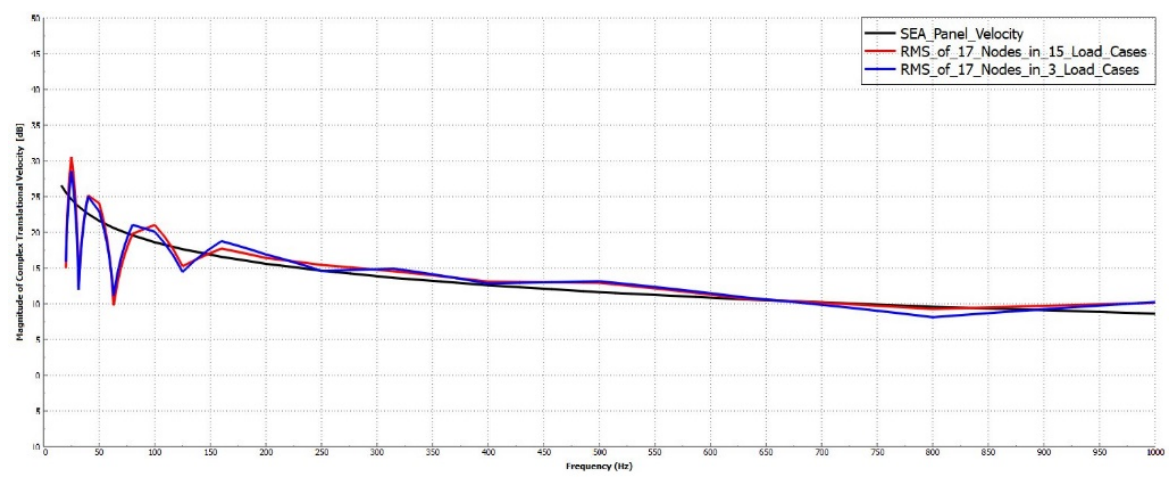

Figure 15. RMS velocity of randomly selected three load cases. Black line: SEA, red line: third-oct. of RMS of all nodes in all load cases, blue line: third-oct. of $R M S$ of all nodes in 3 randomly selected load cases

Good correlation of the results can be observed between the SEA and the Finite Element Method results, regarding both the all-load case averaged and the 3-load case averaged curves. The discrepancies below $150 \mathrm{~Hz}$ are due to the presence of global modes and low modal density, that cannot be considered in SEA. The SEA model is valid over $150 \mathrm{~Hz}$, where the main assumptions prevail.

\section{Conclusions}

An applicable method with high reliability was developed to compare $\mathrm{NVH}$ measurements or Finite Element Simulation with minimal output request to SEA simulation results. It was shown in this study, that in the case of a flat plate, it is adequate to measure the panel velocity in 17 points to obtain a well approximated average response. This number of accelerometers cannot be applied at once in a single run, because of the mass effect, but it is not necessary. It was also shown that at least 3 load cases are required to exclude the influence of the excitation position. Based on the proposed method, 3 different measurement runs should be performed, with different accelerometer positions in each. 


\section{Acknowledgements}

This work was supported by the Hungarian Academy of Sciences and Audi Hungaria Zrt. through the MTA-SZE Lendület Vehicle Acoustics Research Group.

\section{References}

[1] T-M. Kim, J-T. Kim, J-S Kim, SEA-FEM hybrid analysis for predicting Inter-floor impact noise, Applied Acoustics, 129 (2018) pp. 397-407 doi:10.1016/j.apacoust.2017.08.025

[2] R. Gao, Y. Zhang, D. Kennedy, A hybrid boundary element-statistical energy analysis for the mid-frequency vibration of vibro-acoustic systems, Computers and Structures, 203 (2018) pp. 34-42 doi:10.1016/j.compstruc.2018.05.007

[3] G. Borello, L. Gagliardini, Virtual SEA: towards and industrial process, SAE Technical Paper, 2007-01-2302 (2007) doi: $10.4271 / 2007-01-2302$

[4] A. Culla, W. D'Ambrogio, A. Fregolent, S. Milana, Vibroacoustic optimization using a statistical energy analysis model, Journal of Sound and Vibration, 375 (2016) pp. 102-114 doi: $10.1016 / j . j s v .2016 .04 .026$

[5] A. Cicirello, R. S. Langley, L. Kovalevsky, J. Wiidhouse, The Hybrid Finite Element/Statistical Energy Analysis method in "MID-FREQUENCY" - CAE Methodologies for Mid-Frequency Analysis in Vibration and Acoustics, KU Leuven, Leuven, Belgium, 2012, pp. 233-259

[6] M. Brandstetter, Virtual SEA - Mid and high frequency solutions based on standard FE models, 2018 FFT Acoustic Simulation Conference, Toulouse, France, 2018

[7] P. Shorter, V. Cotoni, Statistical Energy Analysis in Engineering Vibroacoustic Analysis: Methods and Applications, John Wiley \& Sons, Ltd., Chichester, West Sussex, United Kingdom, 2016, pp. 339-383

[8] B. J. Schwarz, M. H. Richardson, Experimental modal analysis, CSI Reliability week, Orlando, FL, 35.1 (1999) pp. 1-12.

[9] The Fundamentals of Modal Testing, (2000) Application Note 243-3 Agilent Technologies

[10] N. H. Baharin, R. A. Rahman, Effect of accelerometer mass on thin plate vibration, Jurnal Mekanikal, 29 (2009) pp. 100-111. 